\title{
Stem cell factor improves lung recovery in rats following neonatal hyperoxia-induced lung injury
}

Luis F. Miranda 1,2, Claudia O. Rodrigues ${ }^{3,4}$, Shalini Ramachandran ${ }^{1,2}$, Eneida Torres ${ }^{1,2}$, Jian Huang ${ }^{1,2}$, Jammie Klim², Dorothy Hehre ${ }^{1,2}$, Ian McNiece ${ }^{4}$, Joshua M. Hare ${ }^{4,5}$, Cleide Y. Suguihara ${ }^{1,2}$ and Karen C. Young ${ }^{1,2,4}$

BACKGROUND: Stem cell factor (SCF) and its receptor, c-kit, are modulators of angiogenesis. Neonatal hyperoxia-induced lung injury (HILI) is characterized by disordered angiogenesis. The objective of this study was to determine whether exogenous SCF improves recovery from neonatal HILI by improving angiogenesis.

METHODS: Newborn rats assigned to normoxia (RA: 20.9\% $\mathrm{O}_{2}$ ) or hyperoxia $\left(90 \% \mathrm{O}_{2}\right)$ from postnatal day $(\mathrm{P}) 2$ to 15 , received daily injections of SCF $100 \mu \mathrm{g} / \mathrm{kg}$ or placebo (PL) from P15 to P21. Lung morphometry was performed at P28. Capillary tube formation in SCF-treated hyperoxia-exposed pulmonary microvascular endothelial cells (HPMECs) was determined by Matrigel assay.

RESULTS: As compared with RA, hyperoxic-PL pups had decrease in alveolarization and in lung vascular density, and this was associated with increased right ventricular systolic pressure (RVSP), right ventricular hypertrophy, and vascular remodeling. In contrast, SCF-treated hyperoxic pups had increased angiogenesis, improved alveolarization, and attenuation of pulmonary hypertension as evidenced by decreased RVSP, right ventricular hypertrophy, and vascular remodeling. Moreover, in an in vitro model, SCF increased capillary tube formation in hyperoxia-exposed HPMECs.

CONCLUSION: Exogenous SCF restores alveolar and vascular structure in neonatal rats with HILI by promoting neoangiogenesis. These findings suggest a new strategy to treat lung diseases characterized by dysangiogenesis.

mpaired alveolar development or so-called "alveolar simplification" and dysmorphic vascular growth are the hallmarks of bronchopulmonary dysplasia (BPD) (1). This disease occurs in $\sim 1$ in 3 very-low-birth-weight premature infants and accounts for more than 12,000 new cases in the United States annually (2). Unfortunately, despite a marked improvement in neonatal mortality over the past three decades, the incidence of BPD continues to rise, and the mortality rate for BPD complicated by pulmonary hypertension (PH) remains as high as $70 \%(3)$. For survivors, the health impact is significant; infants with BPD often have prolonged hospital stays and frequently need supplemental oxygen and special services owing to developmental delay and cerebral palsy (1). The economic impact is also tremendous. In 2005, the overall cost of treating BPD in the United States was estimated to be $\sim \$ 2.4$ billion. Thus, effective treatment strategies to counteract this disease are urgently needed. Yet, one of the main problems in developing therapies for BPD is its multifactorial etiology which impacts not only the airways but also the developing microvasculature. The lung circulation in these patients is characterized by dysmorphic vascular growth, including a relative paucity of small pulmonary arteries with an altered pattern of distribution within the interstitium of the distal lung (4). This abnormal vascular development is associated with a downregulation of several angiogenic growth factors (4).

Stem cell factor (SCF), or c-kit ligand, is a glycoprotein which exists in both soluble and membrane bound forms (5). Commonly known to be a mobilizer of stem cells, this ligand is expressed on several cells, including bronchial epithelial (6) and pulmonary microvascular endothelial cells (7). Its receptor, c-kit or CD117, is known to be expressed on diverse populations of cells including endothelial progenitor cells, hematopoietic stem cells, mast cells, fetal lung stromal cells, and putative lung stem cells (8-10). Binding of SCF to c-kit activates several signal transduction pathways that promote angiogenesis, induce cell proliferation, and augment cell sur$\operatorname{vival}(11)$.

Neonatal hyperoxic exposure is a significant contributor to BPD (12). This exposure results in impaired angiogenesis (13), and thus, SCF, a potent proangiogenic factor (14), may be potentially efficacious in alleviating neonatal hyperoxia-induced lung injury (HILI). Indeed, several reports have demonstrated that SCF induces angiogenesis. Matsui et al. (15) demonstrated that SCF promotes angiogenesis in human umbilical vein cells and silencing SCF-inhibited capillary tube formation. Similarly, Fujii et al. (16) demonstrated that administration of SCF improved myocardial function following infarction by promoting neoangiogenesis, and several studies have shown that SCF modulates tumor angiogenesis $(14,17)$. 


\section{Lung reparative effects of SCF Articles}

Interestingly, c-kit, the SCF receptor, has also been shown to be a modulator of angiogenesis (18). This is distinctly plausible, as in prior studies, c-kit mutant mice were shown to have defective angiogenesis following myocardial infarction, and administration of c-kit-positive cells improved myocardial regeneration following injury by promoting angiogenesis (19). Whether harnessing the proangiogenic properties of SCF in neonatal HILI will promote lung repair is unclear. The purpose of this study was to determine whether the administration of SCF would improve angiogenesis and alveolarization in neonatal rats with HILI. We demonstrate that SCF improves alveolarization and vascular density, decreases pulmonary vascular remodeling, and increases lung vascular endothelial growth factor (VEGF) expression in neonatal rats. Moreover, SCF promoted capillary tube formation in hyperoxia-exposed human pulmonary microvascular endothelial cells (HPMECs). These present findings provide new insight into the role of SCF in HILI and suggest a new strategy to decrease the incidence of $\mathrm{BPD}$ in neonates.

\section{RESULTS}

\section{Lung c-Kit and SCF Expression in HILI}

We first sought to ascertain whether hyperoxic exposure would affect the protein expression of SCF and its receptor, c-kit, in the lungs of neonatal pups. Whole lung lysates were obtained from newborn rat pups exposed to normoxia or hyperoxia $\left(90 \% \mathrm{O}_{2}\right)$ for $14 \mathrm{~d}$. The protein expression of c-kit and SCF in lung lysates was determined by western blot. As compared with normoxic pups, there was a twofold decrease in the protein expression of c-kit in lung lysates obtained from hyperoxic pups (Figure 1a). There was however no change in the lung protein expression of SCF following $14 \mathrm{~d}$ of hyperoxia (Figure $1 \mathrm{~b}$ ).

\section{SCF Promotes Angiogenesis in Neonatal Rats With HILI}

We next analyzed the effects of exogenous SCF on lung angiogenesis in neonatal rats with HILI. Neonatal rats exposed to normoxia (RA: $\left.20.9 \% \mathrm{O}_{2}\right)$ or hyperoxia $\left(90 \% \mathrm{O}_{2}\right)$ for $14 \mathrm{~d}$ were randomly assigned to receive daily injections of SCF $100 \mu \mathrm{g} / \mathrm{kg}$ or placebo (PL) from postnatal day (P) 15 to P21. The lungs were removed at P28, and paraffin-embedded lung sections were immunostained with Von Willebrand factor (vWF), an endothelial cell marker. Hyperoxia-exposed rats had a decrease in the number of intra-acinar $\mathrm{vWF}^{\mathrm{pos}}$ vessels/ high-power field (HPF) at P28, similar to that seen in BPD (Figure 2a). This was evidenced by a twofold reduction in vascular density as compared with normoxic rats $(8 \pm 1$ vs. $4 \pm 1$ vessels/HPF; RA-PL vs. hyperoxia-PL; $P<0.001 ; n=$ 5-6/group; Figure 2b). By contrast, administration of SCF to hyperoxia-exposed rats improved vascular density to normoxic levels ( $4 \pm 1$ vs. $9 \pm 1$ vessels/HPF; hyperoxia-PL vs. hyperoxia-SCF; $P<0.001 ; n=5-6 /$ group; Figure 2b). This improvement in vascular density was accompanied by an increase in lung VEGF protein concentration $(400 \pm 13$ vs. $470 \pm 23 \mathrm{pg} / \mathrm{ml}$; hyperoxia-PL vs. hyperoxia-SCF; $P<0.01$; Figure 2c), as determined by enzyme-linked immunosorbent assay.
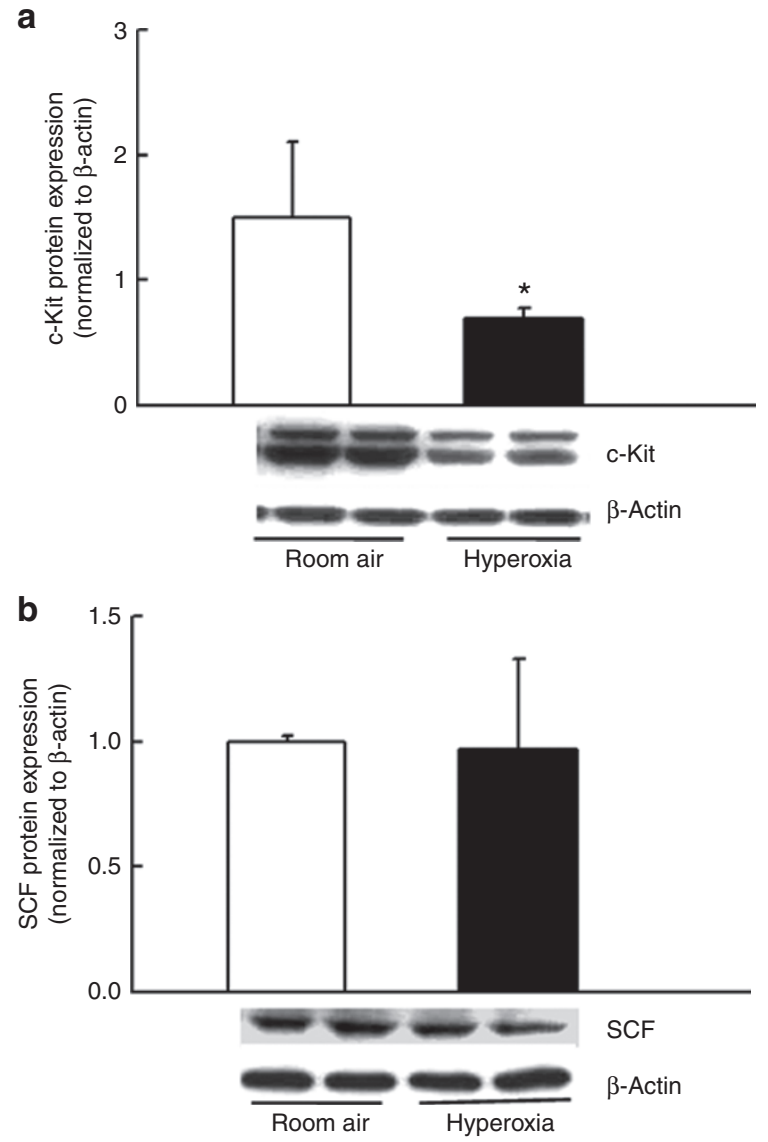

Figure 1. Effect of hyperoxia on lung SCF and c-kit expression. (a) Decreased lung c-kit protein expression in newborn rats exposed to $14 \mathrm{~d}$ of hyperoxia ( ${ }^{*} P<0.05$, room air vs. hyperoxia; $n=5 /$ group). $c$-Kit expression is normalized to $\beta$-actin. A representative western blot is shown in the lower panel. White bars indicate room air, and black bars indicate hyperoxia. (b) Lung SCF expression is not significantly affected by neonatal hyperoxic exposure for $14 \mathrm{~d}$. SCF, stem cell factor.

\section{SCF Increases Capillary Tube Formation in HPMECs}

HPMECs exposed to hyperoxia had decreased number of cordlike structures ( $6.3 \pm 2.1$ vs. $2.6 \pm 0.9$; normoxia vs. hyperoxia; $P<0.001 ; n=6 /$ group; Figure 3a,b). By contrast, hyperoxiaexposed HPMECs treated with SCF showed a dose-dependent increase in the number of cord-like structures (Figure $3 b$ ).

\section{SCF Attenuates PH and Vascular Remodeling in HILI}

$\mathrm{PH}$ in HILI may be secondary to a decrease in vascular density. Thus, we next analyzed whether SCF would improve PH in neonatal rats with HILI. The degree of $\mathrm{PH}$ was evaluated by measuring the right ventricular systolic pressure (RVSP) and the ratio of the weight of the right ventricle to the left ventricle and septum $(\mathrm{RV} / \mathrm{LV}+\mathrm{S})$. Hyperoxia exposed rats had an increase in RVSP $(20 \pm 2$ vs. $37 \pm 6 \mathrm{~mm} \mathrm{Hg}$; RA-PL vs. hyperoxia-PL; $P<0.001 ; n=6-7 /$ group), and in RV/ $\mathrm{LV}+\mathrm{S}(0.27 \pm 0.02$ vs. $0.38 \pm 0.08$; RA-PL vs. hyperoxia-PL; $P$ $<0.001 ; n=6-7$ /group) (Figure 4a,b). By contrast, administration of SCF significantly decreased RVSP ( $37 \pm 6$ vs. $29 \pm 4$ $\mathrm{mm}$ Hg; hyperoxia-PL vs. hyperoxia-SCF; $P<0.001 ; n=6-7 /$ group) and $\mathrm{RV} / \mathrm{LV}+\mathrm{S}(0.38 \pm 0.08$ vs. $0.29 \pm 0.02$; hyperoxia-PL 
a

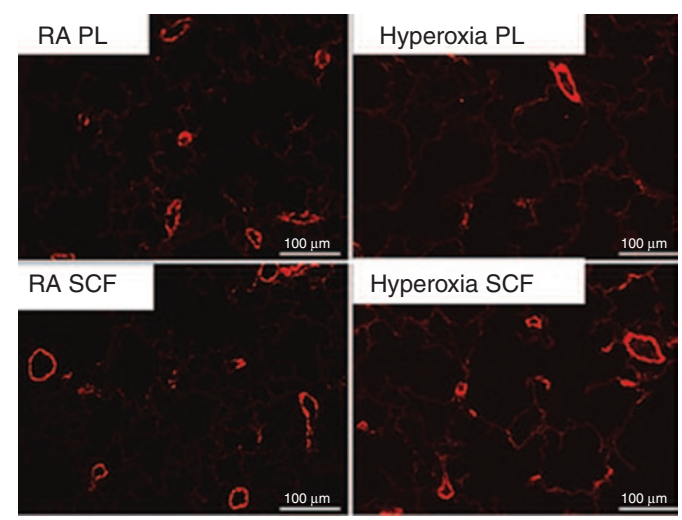

b

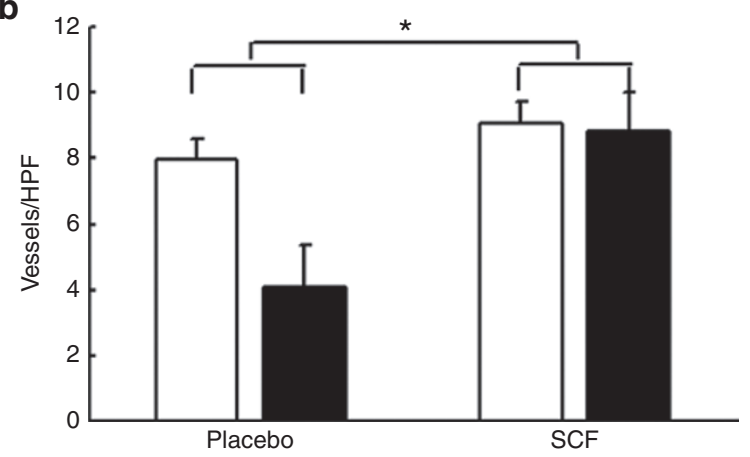

c

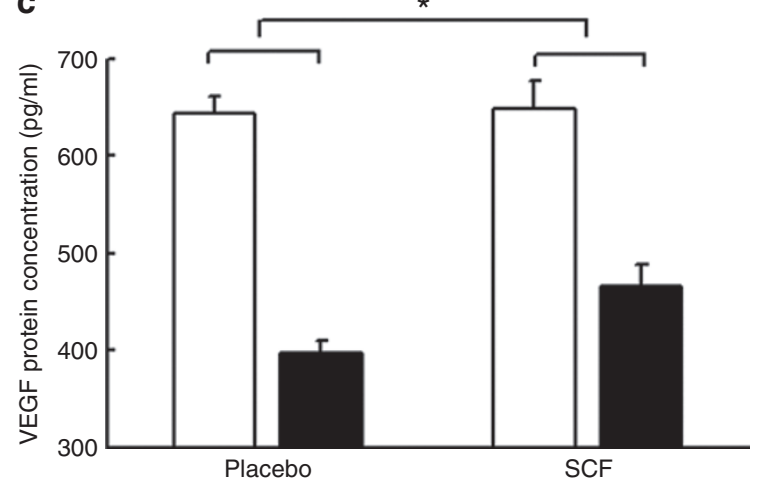

Figure 2. SCF promotes angiogenesis in neonatal rats with HILI. (a) Lung sections stained with vWF (red) demonstrating improved vascular density in the hyperoxia-exposed SCF-treated rats. Original magnification: $\times 100$. Scale bar $=100 \mu \mathrm{m}$. (b) Increased capillary density in the lungs of hyperoxia-exposed SCF-treated animals ( ${ }^{*} P<0.001$, SCF vs. PL; $n=5-6 /$ group). White bars indicate $\mathrm{PL}$, and black bars indicate hyperoxia. (c) Increased VEGF protein synthesis in the lungs of hyperoxia-exposed SCF-treated rats ( ${ }^{*} P<0.01$, SCF vs. $\mathrm{PL} ; n=5-6$ /group). HILI, hyperoxia-induced lung injury; HPF, high-power field; PL, placebo; SCF, stem cell factor; VEGF, vascular endothelial growth factor; vWF, Von Willebrand factor.

vs. hyperoxia-SCF; $P<0.001 ; n=6-7 /$ group) (Figure 4a,b). There was no significant difference in $\mathrm{PH}$ between the normoxia-exposed groups.

Moreover, while hyperoxia exposed rats had an increased vascular remodeling as evidenced by increased medial wall thickening ( $10 \pm 0.6$ vs. $16 \pm 3.3 \%$; RA-PL vs. hyperoxia-PL; $P<$ $0.001 ; n=4-5$ /group), hyperoxic SCF-treated rats had a significant decrease in medial wall thickening ( $16 \pm 3.3$ vs. $12 \pm 3.1 \%$; hyperoxia-PL vs. hyperoxia-SCF; $P<0.001 ; n=4-5$ /group)
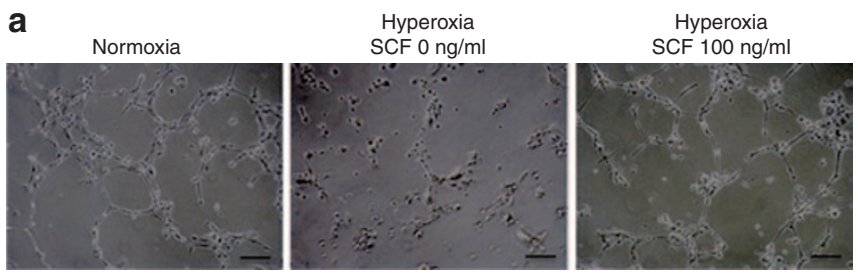

b

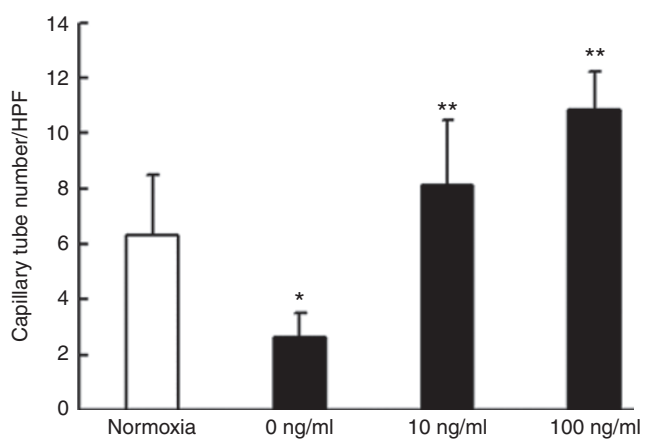

Figure 3. SCF increases capillary tube formation in hyperoxia-exposed HPMECs. (a) Increased tubule formation $20 \mathrm{~h}$ after plating SCF-treated hyperoxia-exposed HPMECs on Matrigel. Original magnification: $\times 100$. Scale bar $=50 \mu \mathrm{m}$. (b). Dose-dependent increase in the number of cordlike structures in the hyperoxia-exposed SCF-treated HPMECs $\left({ }^{*} P<0.04\right.$, normoxia vs. hyperoxia SCF- $0 \mathrm{ng} / \mathrm{ml} ;{ }^{*} P<0.001$, hyperoxia SCF-0 ng/ $\mathrm{ml}$ v. SCF- $10 \mathrm{ng} / \mathrm{ml}$ and SCF- $100 \mathrm{ng} / \mathrm{ml}$ ). White bar represents normoxiaexposed untreated HPMECs, and black bars represent hyperoxia-exposed HPMECs, which were untreated (SCF $0 \mathrm{ng} / \mathrm{ml}$ ) or treated with SCF 10$100 \mathrm{ng} / \mathrm{ml}$. HPF, high-power field; HPMECs, hyperoxia-exposed pulmonary microvascular endothelial cell; SCF, stem cell factor.

(Figure $4 \mathrm{c}, \mathbf{d}$ ). This was associated with decreased lung collagen concentration in the hyperoxia-SCF group ( $4.4 \pm 0.5$ vs. $3.2 \pm 0.2 \mu \mathrm{g}$ collagen/mg protein; hyperoxia-PL vs. hyperoxiaSCF; $P<0.001 ; n=5$-6/group; Figure 4e).

\section{SCF Improves Alveolarization}

Lung angiogenesis is crucial for normal lung alveolarization. Therefore, we next investigated the effects of SCF on lung alveolarization. Mean linear intercept (MLI), lung septal density, and volume fraction of alveoli within parenchymal tissue $\left(\mathrm{V}_{\mathrm{v}}(\mathrm{alv} / \mathrm{par})\right)$ were utilized to assess lung alveolarization. Hyperoxia-exposed rats have decreased alveolarization as evidenced by increased alveolar simplification and decreased septa formation (Figure 5a). Morphometric analysis revealed an increase in MLI ( $38 \pm 4$ vs. $64 \pm 3 \mu \mathrm{m}$; RA-PL vs. hyperoxia-PL; $P<0.001 ; n=6-7$ /group), a decrease in septal density ( $34 \pm 4$ vs. $26 \pm 4$ septa/HPF; RA-PL vs. hyperoxia-PL; $P<0.001 ; n=6-7 /$ group), and an increase in $\mathrm{V}_{\mathrm{v}}(\mathrm{alv} /$ par) in hyperoxia-exposed rats ( $63 \pm 4$ vs. $74 \pm 3 \%$; RA-PL vs. hyperoxia-PL; $P<0.001 ; n=6-7$ /group) (Figure 5b-d). By contrast, administration of SCF decreased MLI (64 \pm 3 vs. $47 \pm 3 \mu \mathrm{m}$; hyperoxia-PL vs. hyperoxia-SCF; $P<0.001 ; n=$ 6-7/group), restored septal density ( $26 \pm 4$ vs. $34 \pm 6$ septa/ HPF; hyperoxia-PL vs. hyperoxia-SCF; $P<0.001 ; n=6-7 /$ group), and reduced $\mathrm{V}_{\mathrm{v}}$ (alv/par) to near-normoxic values (Figure 5b-d). There was no difference in MLI, septal density, or $\mathrm{V}_{\mathrm{v}}(\mathrm{alv} / \mathrm{par})$ between the normoxia-exposed rats. 
a

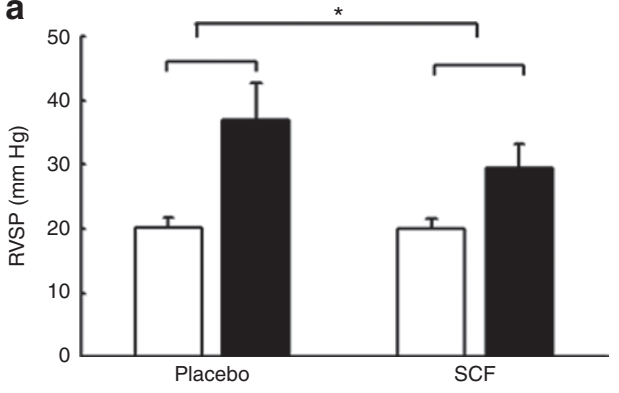

b

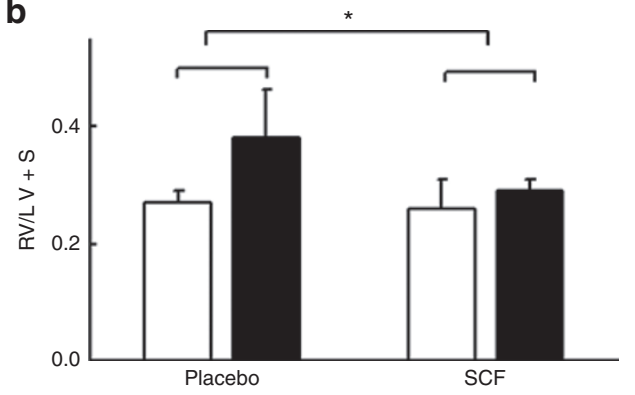

C

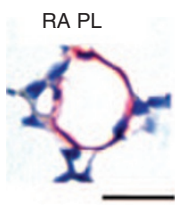
Hyperoxia PL
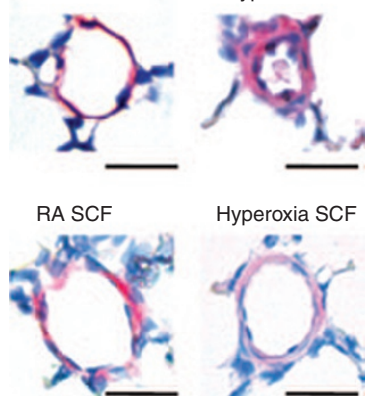

d

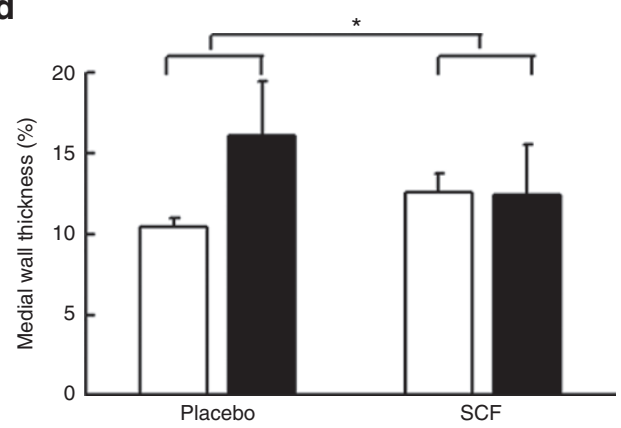

e

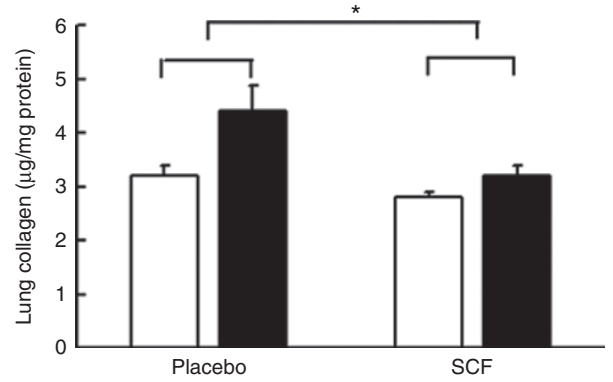

Figure 4. SCF attenuates pulmonary hypertension. (a) Decreased RVSP in the lungs of hyperoxia-exposed SCF-treated rats ${ }^{*} P<0.001, \mathrm{SCF}$ vs. PL; $n=6-7 /$ group). White bars represent $\mathrm{PL}$, and black bars represent hyperoxia. (b) Decreased RV/LV+S in the lungs of hyperoxia-exposed SCF-treated rats $\left({ }^{*} P<0.04\right.$, SCF vs. PL; $n=6-7 /$ group). (c) Decreased $\alpha$-smooth muscle actin immunostaining in the pulmonary arterioles of hyperoxia-exposed SCF-treated rats. Original magnification: $\times 400$. Scale bar: $25 \mu \mathrm{m}$. (d) Decreased medial wall thickness in hyperoxia-exposed SCF-treated rats $(* P<0.001$, SCF vs. PL; $n=5-6 /$ group). (e) Decreased lung collagen in hyperoxia-exposed SCF-treated rats ( ${ }^{*} P<0.001, S C F$ vs. $\left.P L ; n=5-6 / g r o u p\right)$. PL, placebo; $\mathrm{RV} / \mathrm{LV}+\mathrm{S}$, ratio of the weight of the right ventricle to the left ventricle and septum; RVSP, right ventricular systolic pressure; SCF, stem cell factor.

\section{DISCUSSION}

In this study, we show that in a neonatal HILI model, exogenous SCF improves vascular density, attenuates $\mathrm{PH}$, promotes alveolarization, and decreases pulmonary vascular remodeling. Furthermore, we show that SCF promotes capillary tube formation in hyperoxia-exposed pulmonary microvascular endothelial cells. These results suggest that exogenous SCF improves recovery from neonatal HILI by promoting neoangiogenesis, and more importantly, it provides evidence that SCF administration may be a potential therapeutic strategy for neonates with BPD.

SCF is known to be a potent angiogenic factor (17), and previous studies have demonstrated an increase in local SCF expression following organ injury (20). Indeed, Sun et al. demonstrated that SCF mRNA and protein were highly induced in neurons within the zone of injured brain (20). Surprisingly, in our present study, SCF protein expression was not increased in the lungs of neonatal rats with HILI. These findings may imply that an inadequate SCF response following neonatal hyperoxic exposure might be a contributory factor to the impaired neoangiogenesis observed after neonatal HILI. Interestingly, we also demonstrated a twofold decrease in lung c-kit expression following $14 \mathrm{~d}$ of hyperoxic exposure. Although there are no known previously published studies evaluating the expression of c-kit during hyperoxia, this finding is particularly notable as downstream signaling following activation of c-kit by SCF is known to promote the release of several angiogenic factors (21). Moreover, augmentation of SCF/c-kit signaling has been shown to increase capillary tube formation in human umbilical vein endothelial cells (15).

In the present study, we demonstrate that exogenous SCF improves lung angiogenesis in neonatal rats with HILI and furthermore that SCF promotes capillary tube formation in hyperoxia-exposed pulmonary microvascular endothelial cells. This is in keeping with prior studies showing SCF-induced increases in angiogenesis in models of organ injury $(16,22)$ and with published reports showing evidence that SCF increases tumor angiogenesis. In one recent publication, Zhang et al. (17) demonstrated that transfection of rat mammary tumor cells with SCF cDNA resulted in more vascularized tumors. In addition, Sun et al. (14) demonstrated that downregulation of SCF improved survival in orthotopic mouse glioma models by reducing tumor angiogenesis. Furthermore, in an in vitro model, exposure of fatty acid-binding protein 4 -deficient endothelial cells (which have attenuated angiogenic activity) to SCF increased angiogenic sprouting (23).

It should be noted, however, that in our study, the SCFinduced improvement in lung vascular density following HILI was associated with an increase in VEGF concentration. Thus, the improvement in angiogenesis following SCF administration may be a direct effect or potentially an indirect effect by an increase in VEGF release. This modulation of VEGF expression by SCF is in keeping with the findings of several investigators who demonstrated that $\mathrm{SCF} / \mathrm{c}$-kit signaling results in hypoxia-inducible factor- $1 \alpha$-mediated enhancement of VEGF expression in cancer cell lines and hematopoietic stem cells $(21,24)$. 
a

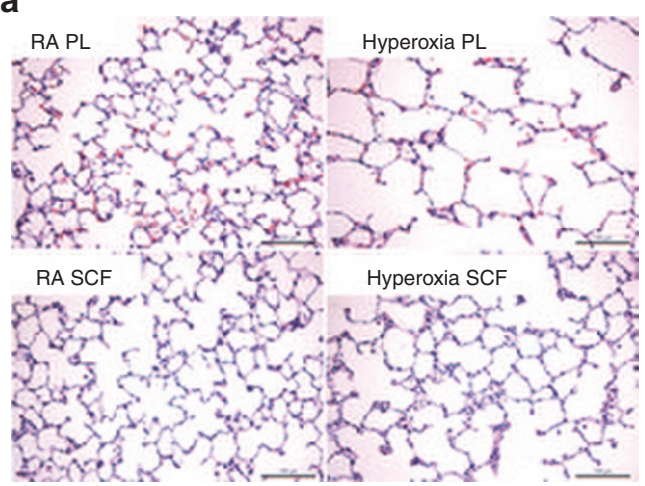

C

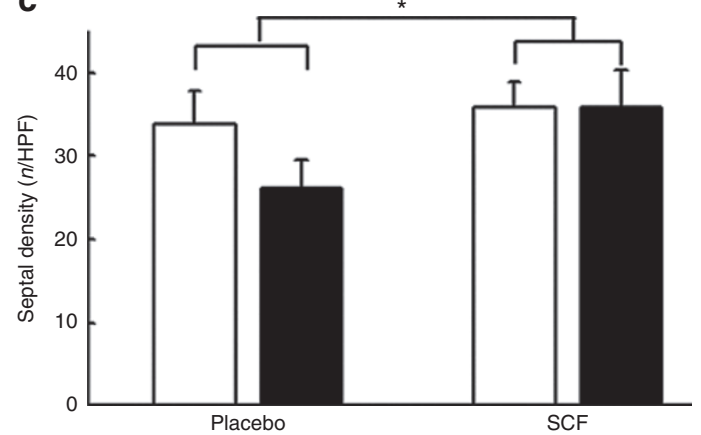

b

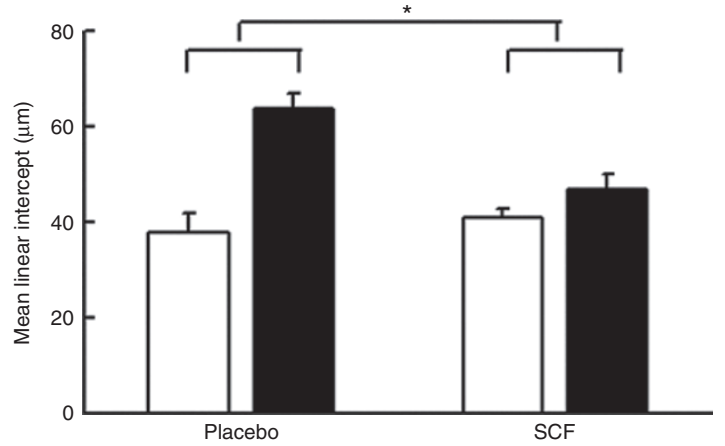

d

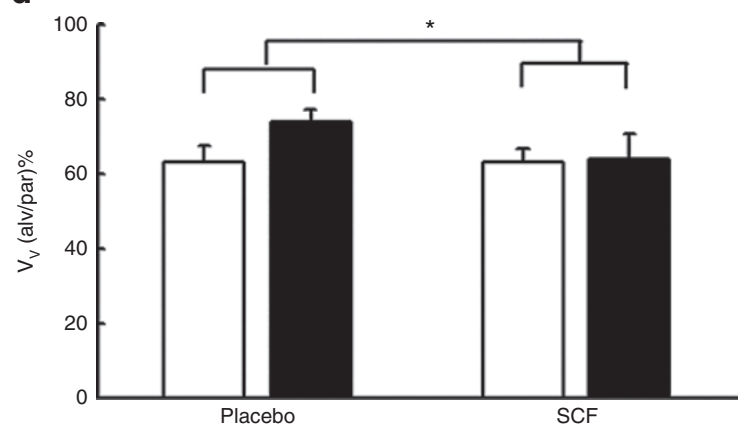

Figure 5. Stem cell factor improves alveolarization. (a) H\&E-stained lung sections demonstrating improved alveolar structure in hyperoxia-exposed SCF-treated rats. Original magnification: $\times 100$. Scale bar: $100 \mu \mathrm{m}$. (b) Decreased mean linear intercept in hyperoxic SCF-treated rats $\left({ }^{*} P<0.001\right.$, SCF vs. PL; $n=6-7 /$ group). White bars represent PL, and black bars represent hyperoxia. (c) Increased septal density in SCF-treated hyperoxic rats ( $P<0.002$, SCF vs. $\mathrm{PL} ; n=6-7 /$ group). (d) Decreased V (alv/par) in SCF-treated hyperoxic rats $\left({ }^{*} P<0.03\right.$, SCF vs. PL; $n=6-7 /$ group). H\&E, hematoxylin and eosin; HPF, highpower field; $\mathrm{PL}$, placebo; $\mathrm{SCF}$, stem cell factor; $\mathrm{V}_{\mathrm{v}}$ (alv/par), volume fraction of alveoli within parenchymal tissue.

In keeping with our findings of improved angiogenesis, we also demonstrate improved $\mathrm{PH}$ in the hyperoxic SCF-treated rats as evidenced by decreased RVSP, right ventricular hypertrophy, and vascular remodeling. This improvement in vascular remodeling was not expected since prior studies have demonstrated that SCF contributes to vascular remodeling in systemic vascular injury models. Wang et al showed that SCF attenuated vascular smooth muscle cell apoptosis and increased intimal hyperplasia after arterial wall injury in a mouse model. Moreover, intimal hyperplasia was significantly decreased in Steel-Dickie mice, which lack the membranebound SCF (25). It is however plausible that the improvement in vascular remodeling seen in our study following SCF administration may be an indirect effect occurring primarily as a consequence of the improvement in lung injury.

Alveolar development and formation of pulmonary blood vessels are closely intertwined processes (4). Arrested alveolarization is a key component of BPD. We showed that SCF administration to hyperoxia-exposed rats promotes an improvement in alveolarization, as evidenced by increased septal density, decreased MLI, and improved $\mathrm{V}_{\mathrm{v}}$ (alv/par). Similar findings have also been shown in other models of organ injury. In a model of liver injury, animals treated with a lethal dose of acetaminophen, and administered with exogenous SCF, had increased survival and reduced serum liver enzymes (26). Similarly, in a mouse model of folic acid-induced kidney injury, administration of SCF to metalloproteinase-9-deficient mice (which have decreased SCF expression) improved renal function and decreased apoptosis in renal intercalated and proximal tubule cells (27).

Finally, it is important to mention the limitations of this study. We utilized a neonatal rodent HILI model as the phenotypic changes evidenced in this model are similar to that seen in BPD. Yet, although hyperoxia is an important contributor to the pathogenesis of BPD, there are several other factors including pre- and postnatal exposure to infection, ventilation, and poor nutrition which contribute to the arrest of lung development evidenced in this disease. It is therefore possible that the additive effects of these insults on the preterm lung may potentially alter the efficacy of this therapy. In addition, although it was not within the scope of our present study to evaluate stem cell migration, another property of SCF, which may be useful in neonatal HILI, is its stem cell mobilization properties. Indeed, the therapeutic efficacy of stem cells in neonatal HILI has been demonstrated in several recent publications, and strategies to increase the mobilization of stem cells to the injured lung have been previously shown to promote lung repair $(28,29)$. Cheng et al. (30) demonstrated that SCF promotes the release of progenitor cells from the bone marrow to areas of injury, and the mobilized cells were shown to contribute to vessel growth. Xiang et al. (22) also showed that cardiomyocyte-specific overexpression of SCF improves myocardial function and 
survival after a myocardial infarction by increasing endothelial progenitor cell recruitment and neovascularization. It is therefore plausible that the increased angiogenesis evidenced in our present study may also be secondary to increased migration of stem cells to the lung. Interestingly, within this context, it should be noted that inhibition of SCF or its receptor, c-kit, was shown to attenuate bleomycin-induced pulmonary fibrosis as well as tumor metastases, and c-kit-positive cells were shown to contribute to $\mathrm{PH}(31-33)$. It is possible that the disparity in our present findings and previous studies may be secondary to the differences in the mechanism of the lung injury, which may impact the fate of stem cells as well as the pleiotropic effects of $\mathrm{SCF} / \mathrm{c}$-kit signaling. Notwithstanding, future studies will need to be performed to evaluate the long-term effects of SCF on the lung and other developing organs. In addition, since other investigators utilizing stem cell-based strategies have demonstrated that experimental BPD may be potentially preventable by early treatment (34), it would be important to explore the efficacy of SCF in preventing BPD.

Nonetheless, our data suggest that exogenous SCF may mediate lung repair following HILI by improving the angiogenic milieu of the lung. We show evidence in vivo and in vitro that SCF promotes neoangiogenesis in neonatal HILI, and this characteristic may provide a new therapeutic strategy for lung repair in preterm infants with BPD.

\section{METHODS}

Animals

Pregnant Sprague Dawley rats were purchased from the Charles River Laboratories (Wilmington, MA). All animals were cared for according to the National Institutes of Health guidelines for the care and use of laboratory animals. All animal experiments were performed according to guidelines set forth by the University of Miami Animal Care and Use Committee.

\section{Neonatal Hyperoxic Model}

Sprague Dawley neonatal rats at P2 $(n=49)$ were randomly assigned to normoxia $\left(20.9 \% \mathrm{O}_{2}\right)$ or hyperoxia $\left(90 \% \mathrm{O}_{2}\right)$ groups from birth to P15. Oxygen exposure was achieved in a Plexiglas chamber by a flowthrough system, and the oxygen level inside the Plexiglas chamber was monitored continuously with Maxtec oxygen analyzers (model: OM25-RME; Maxtec, Salt Lake City, UT). The mothers were switched every $48 \mathrm{~h}$ between the hyperoxic and normoxic chambers to prevent damage to their lung. Litter size was adjusted to 10 pups to control for the effect of litter size on nutrition and growth.

\section{SCF Administration}

At P15, all pups were placed in normoxia and randomly assigned to receive a daily subcutaneous injection of either placebo or polyethylene glycolated SCF ( $100 \mu \mathrm{g} / \mathrm{kg}$; obtained from Ian McNiece, Stem Cell Institute, University of Miami Miller School of Medicine) from P15 to P21. This dose was chosen based on previously reported data (35). Animals were evaluated at P28.

\section{Lung Morphometric Analysis}

Lung perfusion was performed as previously described (36). Serial paraffin-embedded, 5 - $\mu$ m-thick lung sections that were taken from the upper and lower lobes were stained by standard hematoxylin and eosin. Alveolarization was determined by analyzing the septal density, MLI, and $\mathrm{V}_{\mathrm{v}}$ (alv/par) as previously described $(37,38)$. Images from five randomly selected, nonoverlapping parenchymal fields were acquired from lung sections of each animal at $\times 10$ magnification. Care was taken to exclude sections with large bronchioles or vessels. MLI was calculated by determining the average distance between intersects of alveolar septal tissue with a superimposed counting grid.
Septal density was measured by counting the number of secondary septa per HPF. $V_{v}$ (alv/par) was determined by point and intersection counting. Test points were defined as either parenchymal points $\left(\mathrm{P}_{(\mathrm{par})}\right)$ if they hit any gas exchange surface, excluding large blood vessels and bronchi, or alveolar points $\left(\mathrm{P}_{\text {(alv) }}\right)$ if they hit the alveolar lumen. $\mathrm{V}_{\mathrm{v}}$ (alv/par)\% was calculated by the equation $\mathrm{P}_{(\mathrm{alv})} / \mathrm{P}_{(\mathrm{par})}$.

\section{Pulmonary Hypertension}

RVSP was evaluated as previously described (36). RVSP was measured and recorded on a Gould polygraph (model TA-400; Gould Instruments, Cleveland, $\mathrm{OH}$ ). Right ventricular hypertrophy was determined by measuring the ratio of the weight of the right ventricle to the left ventricle and septum.

\section{Pulmonary Vascular Density}

Mid lung sections (5- $\mu \mathrm{m}$ thick)of the upper and lower lobes were deparaffinized, rehydrated, and stained with polyclonal rabbit antihuman vWF (Dako, Carpinteria, CA). Five randomly selected, nonoverlapping parenchymal fields from lung sections of each animal were evaluated. The number of blood vessels $(20-50 \mu \mathrm{m}$ in diameter) in each HPF was quantified by a blinded observer.

\section{Pulmonary Vascular Remodeling}

Paraffin-embedded sections were stained with polyclonal rabbit antihuman vWF (DAKO) and monoclonal mouse anti- $\alpha$-smooth muscle actin ( $\alpha$-sma: 1:500; Sigma-Aldrich, St Louis, MO). Medial wall thickness of partially and fully muscular arteries $(20-50 \mu \mathrm{m})$ was determined by using the formula: $2(\mathrm{MT}) \times 100 / \mathrm{ED}$, where MT is the distance between the internal and external elastic laminas and ED is the external diameter. Approximately 20 randomly chosen arteries were evaluated per slide, and all morphometric analyses were performed by a blinded observer.

\section{Sircol Assay}

Lung homogenates collagen content was quantified using Sircol Assay, which was performed as per the manufacturer's specifications (Biocolor, Carrickfergus, UK).

\section{Western Blot Analysis}

The lungs of neonatal rat pups exposed to hyperoxia or normoxia for $2 \mathrm{wk}$ were dissected as previously described (36). Western blot analysis was performed as previously described (36). The primary antibodies utilized were: polyclonal rabbit antihuman c-kit antibody (DAKO) and polyclonal goat antimouse SCF antibody (Santa Cruz Biotechnology, Santa Cruz, CA).

\section{VEGF Concentration}

VEGF protein concentration in lung homogenates was determined using a Quantikine ELISA kit (R\&D Systems, Minneapolis, MN), as per the manufacturer's specifications.

\section{Matrigel Assay}

The effect of SCF on capillary tube formation in vitro was determined by Matrigel assay as previously described (39). HPMECs (Lonza, Allendale, NJ) were cultured to passages 3-6, plated in $100-\mathrm{mm}$ dishes, and serum starved for $48 \mathrm{~h}$. Serum-starved HPMECs were treated with varying doses of SCF $(0-100 \mathrm{ng} / \mathrm{ml})$ and cultured in normoxic $(20.9 \%$ $\mathrm{O}_{2}$ and $\left.5 \% \mathrm{CO}_{2}\right)$ or hyperoxic $\left(95 \% \mathrm{O}_{2}\right.$ and $\left.5 \% \mathrm{CO}_{2}\right)$ conditions for $72 \mathrm{~h}$. Capillary tube formation was assessed on growth factor-reduced Matrigel-coated wells (BD Biosciences, San Diego, CA). Bright field images were collected at 5 and $20 \mathrm{~h}$. All experiments were performed in triplicate, and tube formation was quantified by measuring the number of capillary-like structures in at least three HPFs per well.

\section{Statistics}

Results are reported as mean \pm SD. Data were analyzed by two-way ANOVA followed by a post hoc analysis (Holm-Sidak analysis). Values of $\mathrm{P}<0.05$ were considered statistically significant. Statistical analysis was performed using SigmaStat software (SyStat Software, San Jose, CA).

\section{STATEMENT OF FINANCIAL SUPPORT}

The financial support of Ikaria Advancing Newborn Medicine Grant program to L.F.M., Florida Biomedical Research Award to K.Y., National Institute of 
Health Career Development Award to K.Y., Batchelor Foundation Award to K.Y., and University of Miami Project New Born is acknowledged.

Disclosure: There are no disclosures.

\section{REFERENCES}

1. Jobe AH, Bancalari E. Bronchopulmonary dysplasia. Am J Respir Crit Care Med 2001;163:1723-9.

2. Baraldi E, Filippone M. Chronic lung disease after premature birth. N Engl J Med 2007;357:1946-55.

3. Khemani E, McElhinney DB, Rhein L, et al. Pulmonary artery hypertension in formerly premature infants with bronchopulmonary dysplasia: clinical features and outcomes in the surfactant era. Pediatrics 2007;120:1260-9.

4. Thébaud B, Abman SH. Bronchopulmonary dysplasia: where have all the vessels gone? Roles of angiogenic growth factors in chronic lung disease. Am J Respir Crit Care Med 2007;175:978-85.

5. Anderson DM, Williams DE, Tushinski R, et al. Alternate splicing of mRNAs encoding human mast cell growth factor and localization of the gene to chromosome 12q22-q24. Cell Growth Differ 1991;2:373-8.

6. Wen LP, Fahrni JA, Matsui S, Rosen GD. Airway epithelial cells produce stem cell factor. Biochim Biophys Acta 1996;1314:183-6.

7. Heinrich MC, Dooley DC, Freed AC, et al. Constitutive expression of steel factor gene by human stromal cells. Blood 1993;82:771-83.

8. Kajstura J, Rota M, Hall SR, et al. Evidence for human lung stem cells. $\mathrm{N}$ Engl J Med 2011;364:1795-806.

9. Lindsey JY, Ganguly K, Brass DM, et al. c-Kit is essential for alveolar maintenance and protection from emphysema-like disease in mice. Am J Respir Crit Care Med 2011;183:1644-52.

10. Papayannopoulou T, Brice M, Broudy VC, Zsebo KM. Isolation of c-kit receptor-expressing cells from bone marrow, peripheral blood, and fetal liver: functional properties and composite antigenic profile. Blood 1991;78:1403-12.

11. Lennartsson J, Rönnstrand L. Stem cell factor receptor/c-Kit: from basic science to clinical implications. Physiol Rev 2012;92:1619-49.

12. Bonikos DS, Bensch KG, Ludwin SK, Northway WH Jr. Oxygen toxicity in the newborn. The effect of prolonged 100 per cent $\mathrm{O} 2$ exposure on the lungs of newborn mice. Lab Invest 1975;32:619-35.

13. Shaffer SG, O'Neill D, Bradt SK, Thibeault DW. Chronic vascular pulmonary dysplasia associated with neonatal hyperoxia exposure in the rat. Pediatr Res 1987;21:14-20.

14. Sun L, Hui AM, Su Q, et al. Neuronal and glioma-derived stem cell factor induces angiogenesis within the brain. Cancer Cell 2006;9:287-300.

15. Matsui J, Wakabayashi T, Asada M, Yoshimatsu K, Okada M. Stem cell factor/c-kit signaling promotes the survival, migration, and capillary tube formation of human umbilical vein endothelial cells. J Biol Chem 2004;279:18600-7.

16. Fujii $\mathrm{H}$, Sun Z, Li SH, et al. Ultrasound-targeted gene delivery induces angiogenesis after a myocardial infarction in mice. JACC Cardiovasc Imaging 2009;2:869-79.

17. Zhang W, Stoica G, Tasca SI, Kelly KA, Meininger CJ. Modulation of tumor angiogenesis by stem cell factor. Cancer Res 2000;60:6757-62.

18. Takakura N, Watanabe T, Suenobu S, et al. A role for hematopoietic stem cells in promoting angiogenesis. Cell 2000;102:199-209.

19. Fazel S, Cimini M, Chen L, et al. Cardioprotective c-kit+ cells are from the bone marrow and regulate the myocardial balance of angiogenic cytokines. J Clin Invest 2006;116:1865-77.

20. Sun L, Lee J, Fine HA. Neuronally expressed stem cell factor induces neural stem cell migration to areas of brain injury. J Clin Invest 2004;113: 1364-74.
21. Litz J, Krystal GW. Imatinib inhibits c-Kit-induced hypoxia-inducible factor-1alpha activity and vascular endothelial growth factor expression in small cell lung cancer cells. Mol Cancer Ther 2006;5:1415-22.

22. Xiang FL, Lu X, Hammoud L, et al. Cardiomyocyte-specific overexpression of human stem cell factor improves cardiac function and survival after myocardial infarction in mice. Circulation 2009;120:1065-74, 9 p following 1074 .

23. Elmasri H, Ghelfi E, Yu CW, et al. Endothelial cell-fatty acid binding protein 4 promotes angiogenesis: role of stem cell factor/c-kit pathway. Angiogenesis 2012;15:457-68.

24. Pedersen M, Löfstedt T, Sun J, Holmquist-Mengelbier L, Påhlman S, Rönnstrand L. Stem cell factor induces HIF-1alpha at normoxia in hematopoietic cells. Biochem Biophys Res Commun 2008;377:98-103.

25. Wang CH, Anderson N, Li SH, et al. Stem cell factor deficiency is vasculoprotective: unraveling a new therapeutic potential of imatinib mesylate. Circ Res 2006;99:617-25.

26. Simpson K, Hogaboam CM, Kunkel SL, Harrison DJ, Bone-Larson C, Lukacs NW. Stem cell factor attenuates liver damage in a murine model of acetaminophen-induced hepatic injury. Lab Invest 2003;83:199-206.

27. Bengatta S, Arnould C, Letavernier E, et al. MMP9 and SCF protect from apoptosis in acute kidney injury. J Am Soc Nephrol 2009;20:787-97.

28. Jungraithmayr W, De Meester I, Matheeussen V, Baerts L, Arni S, Weder W. CD26/DPP-4 inhibition recruits regenerative stem cells via stromal cellderived factor- 1 and beneficially influences ischaemia-reperfusion injury in mouse lung transplantation. Eur J Cardiothorac Surg 2012;41:1166-73.

29. Sutsko RP, Young KC, Ribeiro A, et al. Long-term reparative effects of mesenchymal stem cell therapy following neonatal hyperoxia-induced lung injury. Pediatr Res 2013;73:46-53.

30. Cheng M, Zhou J, Wu M, et al. CXCR4-mediated bone marrow progenitor cell maintenance and mobilization are modulated by c-kit activity. Circ Res 2010;107:1083-93.

31. Aono $Y$, Nishioka $Y$, Inayama $M$, et al. Imatinib as a novel antifibrotic agent in bleomycin-induced pulmonary fibrosis in mice. Am J Respir Crit Care Med 2005;171:1279-85.

32. Gambaryan N, Perros F, Montani D, Cohen-Kaminsky S, Mazmanian GM, Humbert M. Imatinib inhibits bone marrow-derived c-kit+ cell mobilisation in hypoxic pulmonary hypertension. Eur Respir J 2010;36:1209-11.

33. Kuonen F, Laurent J, Secondini C, et al. Inhibition of the Kit ligand/c-Kit axis attenuates metastasis in a mouse model mimicking local breast cancer relapse after radiotherapy. Clin Cancer Res 2012;18:4365-74.

34. Chang YS, Choi SJ, Ahn SY, et al. Timing of umbilical cord blood derived mesenchymal stem cells transplantation determines therapeutic efficacy in the neonatal hyperoxic lung injury. PLoS ONE 2013;8:e52419.

35. Norol F, Merlet P, Isnard R, et al. Influence of mobilized stem cells on myocardial infarct repair in a nonhuman primate model. Blood 2003;102:43618.

36. Young KC, Torres E, Hatzistergos KE, Hehre D, Suguihara C, Hare JM. Inhibition of the SDF-1/CXCR4 axis attenuates neonatal hypoxia-induced pulmonary hypertension. Circ Res 2009;104:1293-301.

37. Knudsen L, Weibel ER, Gundersen HJ, Weinstein FV, Ochs M. Assessment of air space size characteristics by intercept (chord) measurement: an accurate and efficient stereological approach. J Appl Physiol 2010;108:412-21.

38. Ochs M, Nyengaard JR, Jung A, et al. The number of alveoli in the human lung. Am J Respir Crit Care Med 2004;169:120-4.

39. Thébaud B, Ladha F, Michelakis ED, et al. Vascular endothelial growth factor gene therapy increases survival, promotes lung angiogenesis, and prevents alveolar damage in hyperoxia-induced lung injury: evidence that angiogenesis participates in alveolarization. Circulation 2005;112:2477-86. 